\title{
Review: no evidence exists that antidepressants acting at $>1$ pharmacological site differ in efficacy from SSRIs in major depression
}

\author{
Freemantle N, Anderson IM, Young P. Predictive value of pharmacological activity for the relative efficacy of \\ antidepressant drugs. Meta-regression analysis. Br J Psychiatry 2000 Oct;177:292-302.
}
QUESTION: When treating major depression, are there specific pharmacological characteristics of alternative antidepressants that alter their efficacy compared with that of selective serotonin reuptake inhibitors (SSRIs)?

\section{Data sources}

Studies were identified by searching Medline (1966-97) and EMBASE/Excerpta Medica (1974-97), reviewing the reference lists of identified articles, and examining existing bibliographies and reviews.

\section{Study selection}

Studies were selected if they were randomised, double blind, controlled trials comparing the effectiveness of an SSRI with an alternative antidepressant drug (one that had a primary effect on 5-HT and/or noradrenaline reuptake and/or $5-\mathrm{HT}_{2}$ antagonism) in adult or elderly patients with major depression.

\section{Data extraction}

Data were extracted on the main outcome measure, scores on the Hamilton Depression Rating Scale (HDRS) but where not available scores on the Montgomery Asberg Depression Rating Scale or the Clinical Global Impression Scale were used. Standardised effect sizes were estimated from the data.

\section{Main results}

105 trials met the selection criteria involving 11537 patients, 5937 treated with an SSRI (fluoxetine was the most common), and 5600 treated with an alternative (amitriptyline was the most common). A metaregression technique was used to examine the extent to which individual factors (including specific pharmacological properties [dual action, triple action, noradrenaline reuptake, 5-HT reuptake, and 5- $\mathrm{HT}_{2}$ antagonism], treatment setting, dose of comparator, method of analysis, age of patients, measurement scale used, and sponsor of trial) predicted a positive outcome. None of the factors had a statistically significant predictive effect on outcome. The table shows the coefficients for some of the pharmacological properties.

Source of funding:

Wyeth Laboratories,

$U K$.

For correspondence:

Professor $N$

Freemantle,

Department of Primary

Care and General

Practice, University of

Birmingham,

Edgbaston,

Birmingham B15 2TT,

UK. Fax $+44(0) 121$

4146571 .

\section{Conclusion}

No evidence exists to support that antidepressants acting at more than 1 pharmacological site differ in efficacy from drugs selective for serotonin reuptake in the treatment of major depression.

Predictive effects of pharmacological action

\begin{tabular}{ll} 
Covariate & Coefficient $(95 \% \mathrm{Cl})$ \\
\hline 5-HT reuptake inhibition & $-0.003(-0.064$ to 0.048$)$ \\
\hline Noradrenaline reuptake & $0.006(-0.042$ to 0.082$)$ \\
\hline $5-\mathrm{HT}_{2}$ antagonism & $-0.001(-0.060$ to 0.055$)$ \\
\hline
\end{tabular}

\section{COMMENTARY}

The introduction of SSRIs led to a debate as to whether they were as effective as older, less pharmacologically selective tricyclic antidepressants (TCAs) in severe depression. ${ }^{1}$ Marketing of new antidepressants, particularly venlafaxine and mirtazapine, ingeniously captured this concern by suggesting that drugs which enhance both 5-HT and noradrenaline neurotransmission could be more effective than SSRIs Freemantle et al, however, in their meta-regression of randomised studies fail to find any correlation between "dual" pharmacological activity and therapeutic efficacy. In addition they find no evidence that concomitant 5-HT, receptor blockade offers advantages.

The finding of this study is not surprising, partly because clinical differences between antidepressants may be apparent only in certain populations, such as the severely ill. More importantly, the analysis requires a better knowledge than we currently possess about which pharmacological properties of antidepressants are truly relevant to their therapeutic efficacy. In addition, what we do know is open to debate. For example, the TCA desipramine, classically regarded as a selective noradrenaline reuptake blocker, inhibits 5-HT reuptake in vivo and probably also antagonises $5-\mathrm{HT}_{2}$ receptors. ${ }^{2}$ Should we therefore think of it as "dual" or even "triple acting"? Both imipramine and clomipramine can be regarded as "dual acting" (and are by Freemantle $e t$ al) but the superior efficacy of clomipramine in obsessive compulsive disorder is not explained by this classification. Important clinical distinctions among antidepressants probably reflect quantitative rather than qualitative pharmacological differences as well as the precise balance among these various properties.

As a psychopharmacologist I am interested in the differential pharmacology of the antidepressant I use, but as a clinician what I would most like to know is whether any of the individual antidepressants in this analysis (regardless of its purported pharmacology) is more effective than SSRIs. For example, is amitriptyline, often considered to be the "best" TCA, superior to SSRIs? What about venlafaxine? As it stands, like FE Smith's judge, we are better informed, but none the wiser.

Phil Cowen, MD Warneford Hospital Oxford, UK

1 Amsterdam JD. Selective serotonin reuptake inhibitor efficacy in severe and melancholic depression.J Psychopharmacol 1998;12:S99-111.

2 Cowen PJ, Geaney DP, Schachter M, et al. Desipramine treatment in normal subjects. Effects on neuroendocrine responses to tryptophan and on platelet serotonin (5-HT) related receptors. Arch Gen Psychiatry 1986;43:61-7.

3 Anderson IM. Selective serotonin reuptake inhibitors versus tricyclic antidepressants: a meta-analysis of efficacy and tolerability.J Affect Disord 2000;58:19-36. 